Supplementary Information

\title{
Double-Net Enclosed Sulfur Composite as A New Cathode in Lithium Sulfur Batteries
}

\author{
Yingying Lv, Mingwei Shang, Xi Chen, Junjie Niu* \\ Department of Materials Science and Engineering, CEAS, University of Wisconsin- \\ Milwaukee Milwaukee, WI 53211, USA. \\ *Email: niu@uwm.edu
}

\section{This file includes:}

Figures S1 to S10 with legends

Table S1

References cited 

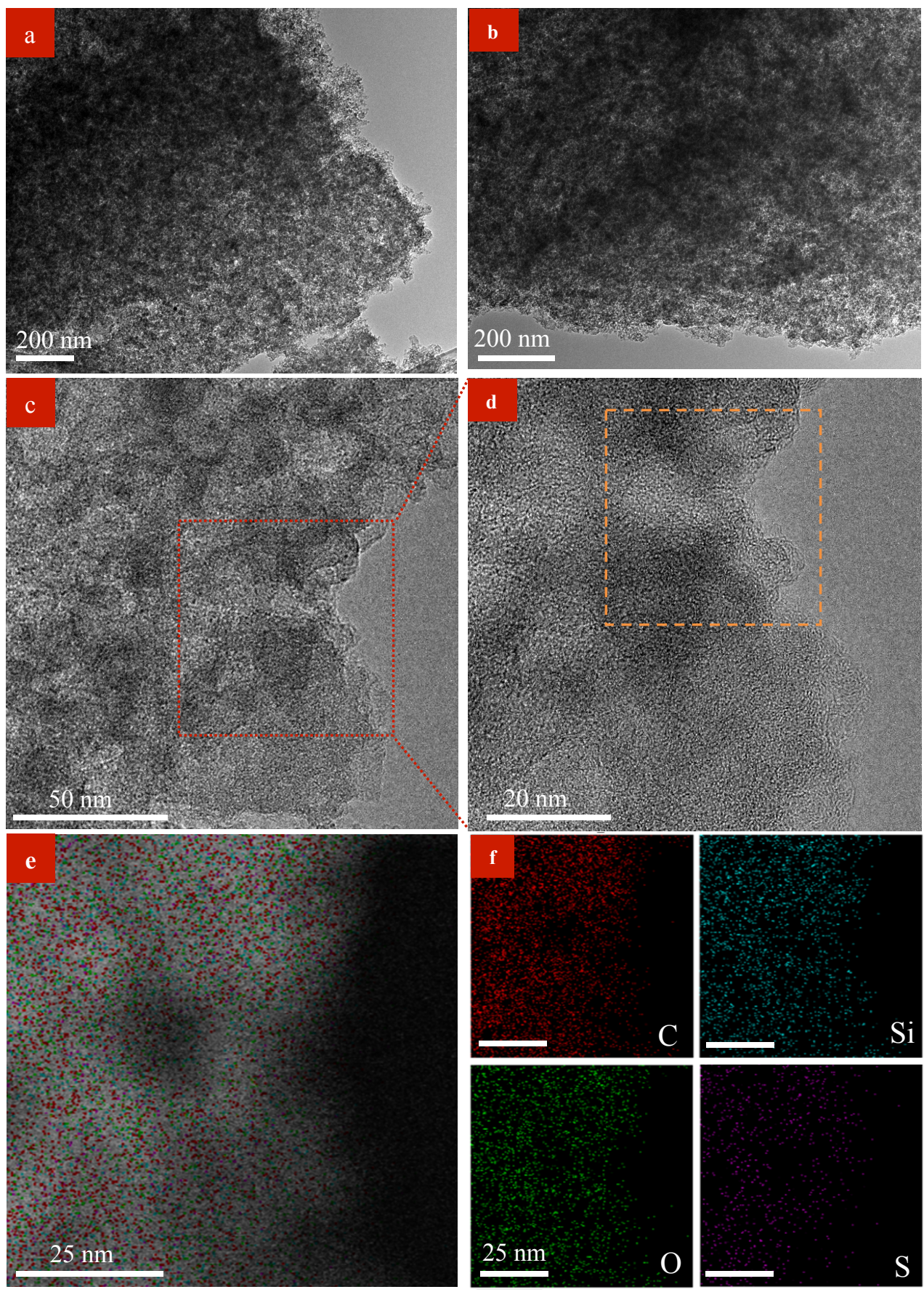

Figure S1. TEM images with low (a-b) and high (c-d) magnifications and the corresponding elemental mappings (e-f) of the $\mathrm{S} @ \mathrm{SiO}_{2} @ \mathrm{AC}$ composite. 

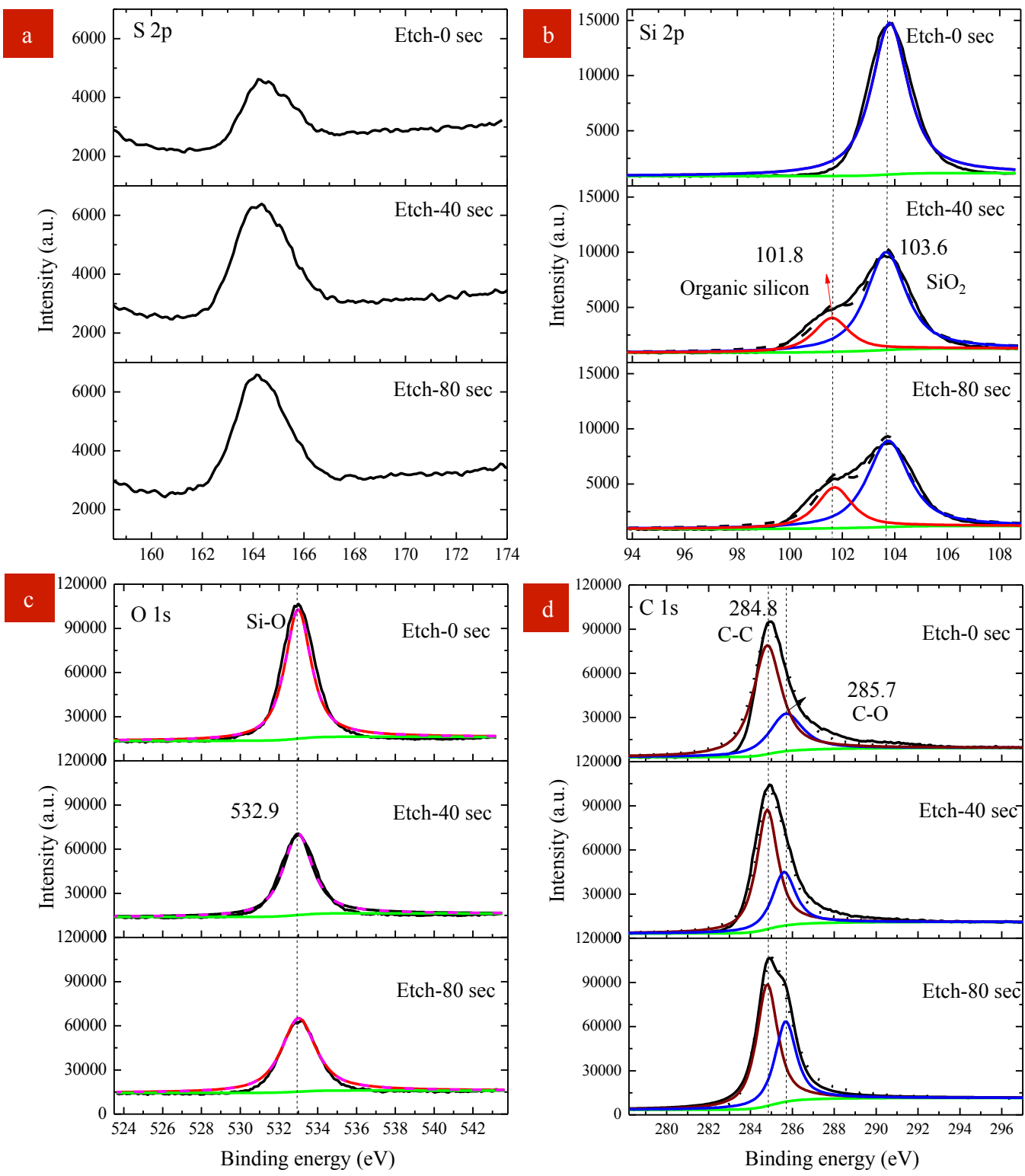

Figure S2. XPS depth profiling of as-received $\mathrm{S} @ \mathrm{SiO}_{2} @ \mathrm{AC}$ composite upon etching $0 \mathrm{sec}, 40 \mathrm{sec}$, and $80 \mathrm{sec}$. The binding energy evolution of S2p (a), $\mathrm{Si} 2 \mathrm{p}(\mathrm{b}), \mathrm{O} 1 \mathrm{~s}(\mathrm{c})$, and C1s (d), respectively. 


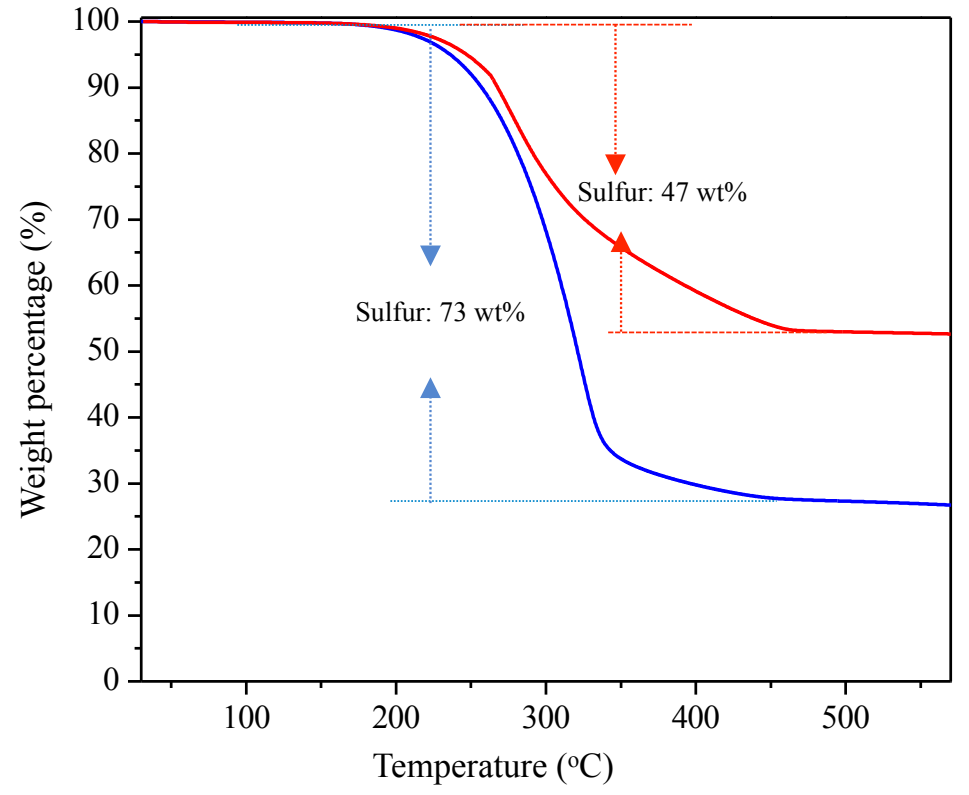

Figure S3. TGA data of the double-net composites with high sulfur loadings of $73 \mathrm{wt} \%$ and $47 \mathrm{wt} \%$ under Ar, respectively. 

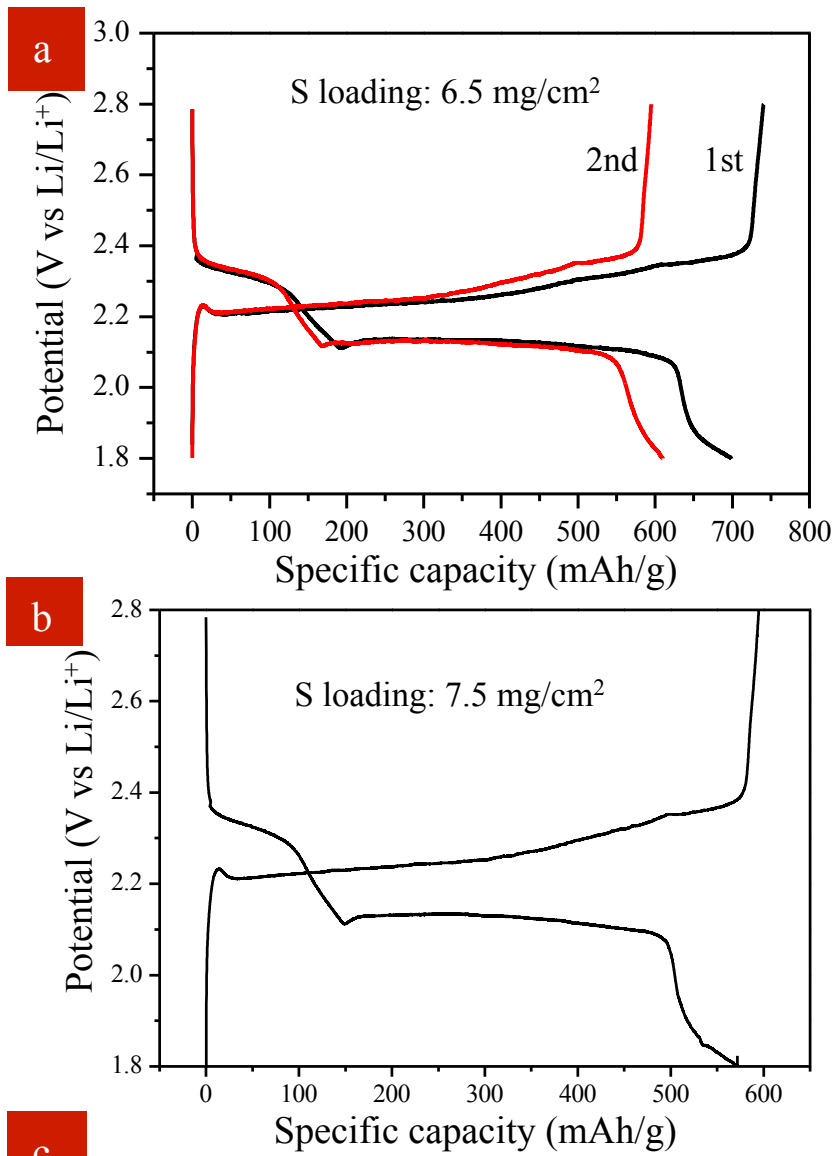

c

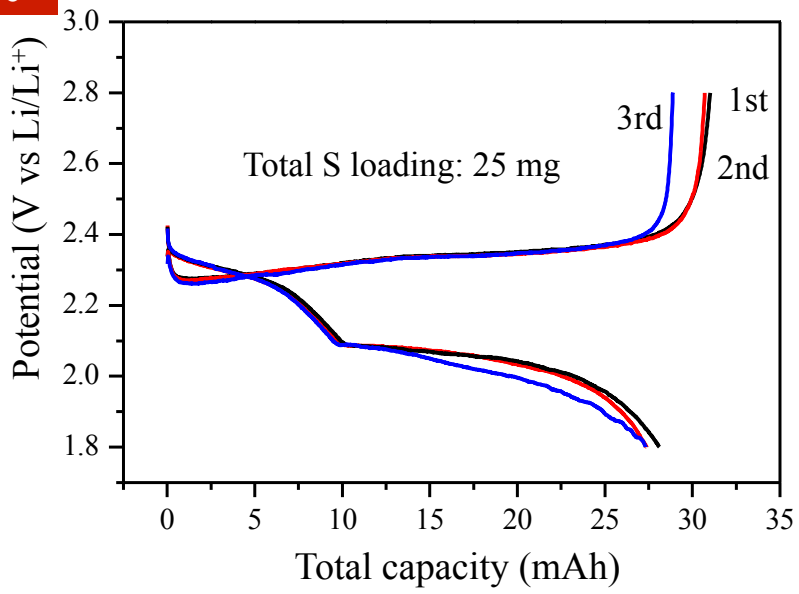

Figure S4. Charge/discharge profile of the battery using $\mathrm{S} @ \mathrm{SiO}_{2} @ \mathrm{AC}$ composite as cathode with sulfur loadings of $\sim 6.5 \mathrm{mg} /$ $\mathrm{cm}^{2}$ (a) and $7.5 \mathrm{mg} / \mathrm{cm}^{2}$ (b) at a rate of $0.1 \mathrm{C}$, respectively. Pouch cell performance with a total sulfur loading of $25 \mathrm{mg}$ at $0.1 \mathrm{C}$ (c). 


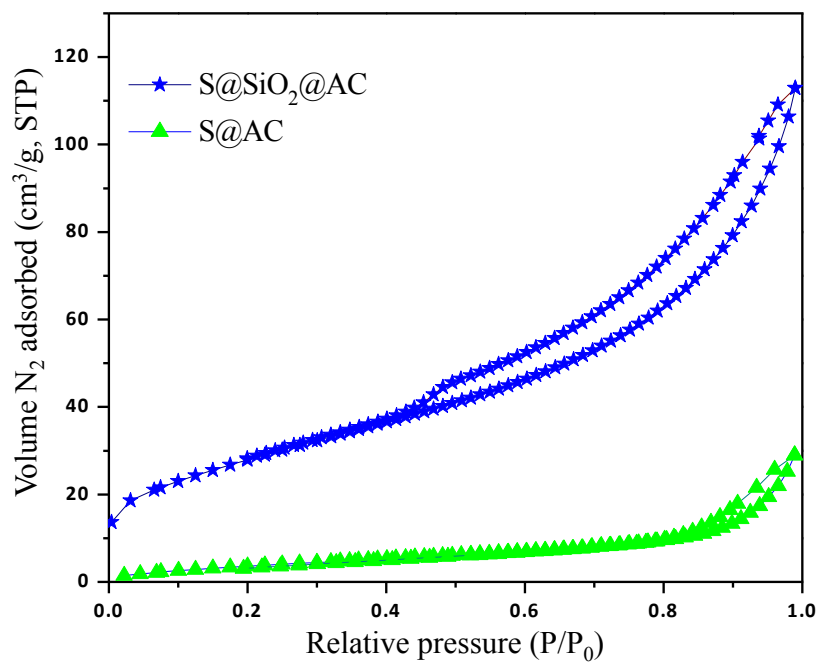

\begin{tabular}{|l|c|c|}
\hline & $\begin{array}{c}\text { Surface area } \\
\left(\mathrm{m}^{2} / \mathrm{g}\right)\end{array}$ & $\begin{array}{c}\text { Pore volume } \\
\left(\mathrm{cm}^{3} / \mathrm{g}\right)\end{array}$ \\
\hline $\mathrm{S} @ \mathrm{SiO}_{2} @ \mathrm{AC}$ & 100 & 0.17 \\
\hline $\mathrm{S} @ \mathrm{AC}$ & 14.9 & 0.04 \\
\hline
\end{tabular}

Figure S5. Nitrogen sorption-desorption isotherms of the S@AC and $\mathrm{S} @ \mathrm{SiO}_{2} @ \mathrm{AC}$ composites (47 wt\% S). 

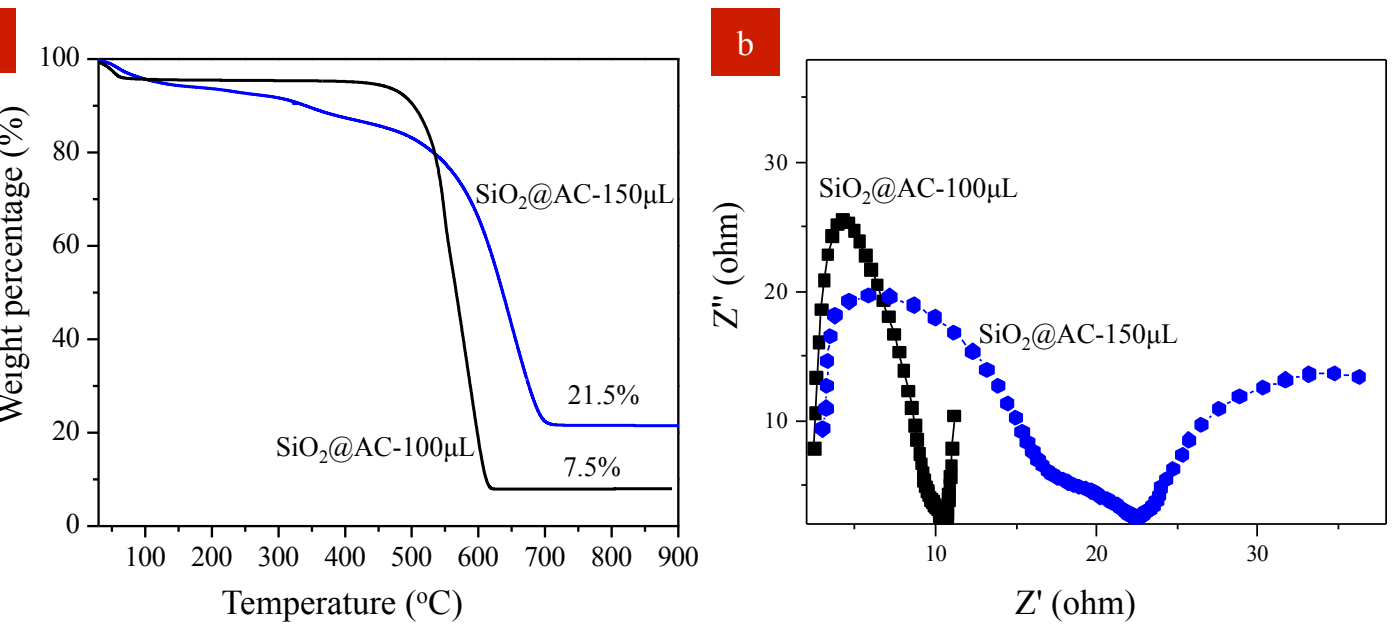

Figure S6. Thermal and electrochemical analysis of the $\mathrm{SiO}_{2} @ \mathrm{AC}$ double-net composites with different TEOS amounts. (a) TGA data of $\mathrm{SiO}_{2} @ \mathrm{AC}-100 \mu \mathrm{L}$ and $\mathrm{SiO}_{2} @ \mathrm{AC}-150 \mu \mathrm{L}$ composites under air. (b) The Nyquist plots of $\mathrm{SiO}_{2} @ \mathrm{AC}-100 \mu \mathrm{L}$ and $\mathrm{SiO}_{2} @ \mathrm{AC}-150 \mu \mathrm{L}$ composites at $5^{\text {th }}$ cycle at $0.2 \mathrm{C}$ obtained from the EIS measurements. 

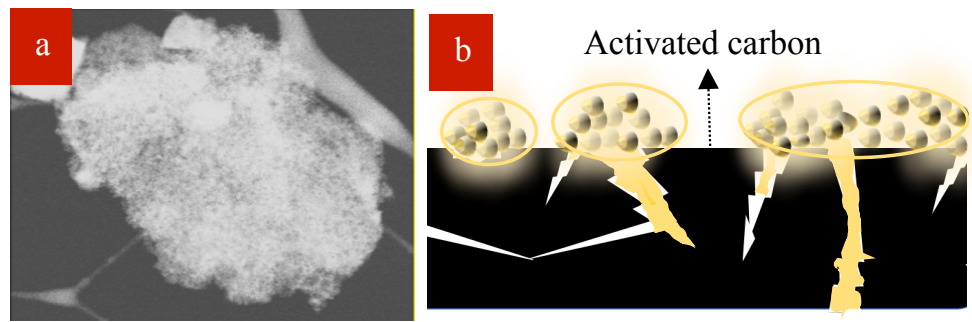

Aggregated sulfur
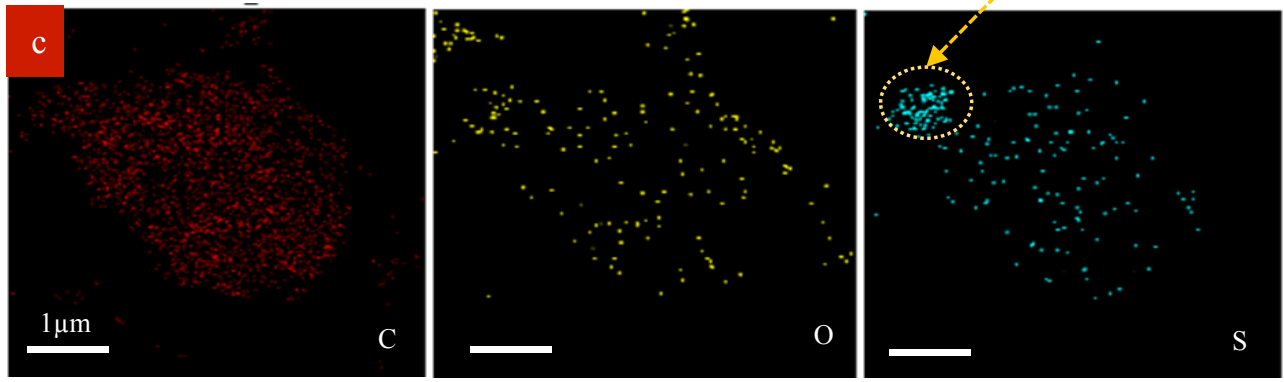

Figure S7. Chemical composition of the S@AC composite. TEM image (a) and the corresponding elemental mappings (c). (b)

Schematic graph of the sulfur aggregation on the surface. 

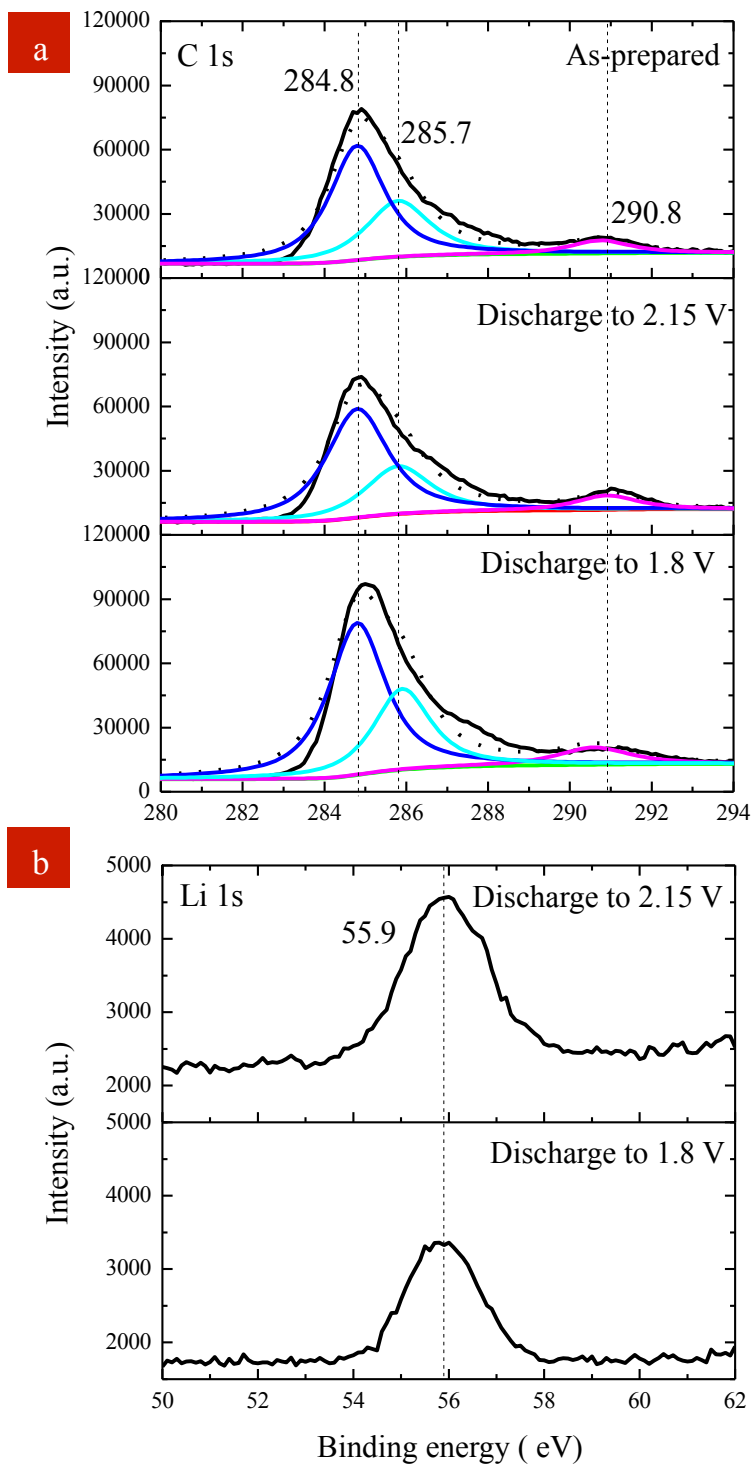

Figure S8. C1s (a) and Li 1s (b) spectra of the $\mathrm{S} @ \mathrm{SiO}_{2} @ \mathrm{AC}$ composite upon different discharge states. 

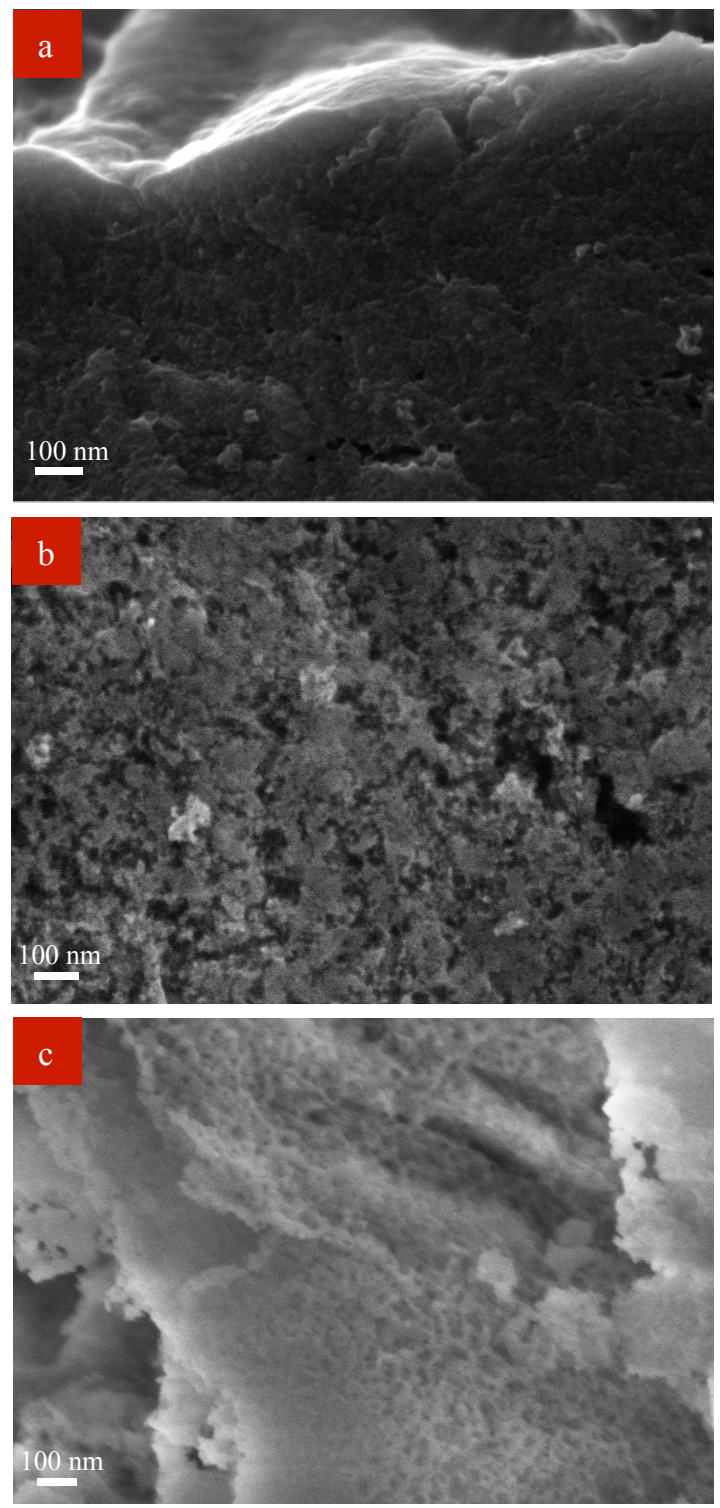

Figure S9. SEM images of (a) AC, (b) $\mathrm{SiO}_{2}$ @ AC composite, and (c) the pure $\mathrm{SiO}_{2}$ after removing the $\mathrm{AC}$ from $\mathrm{SiO}_{2} @ \mathrm{AC}$ composite at $800{ }^{\circ} \mathrm{C}$ in the air for $1 \mathrm{~h}$. 

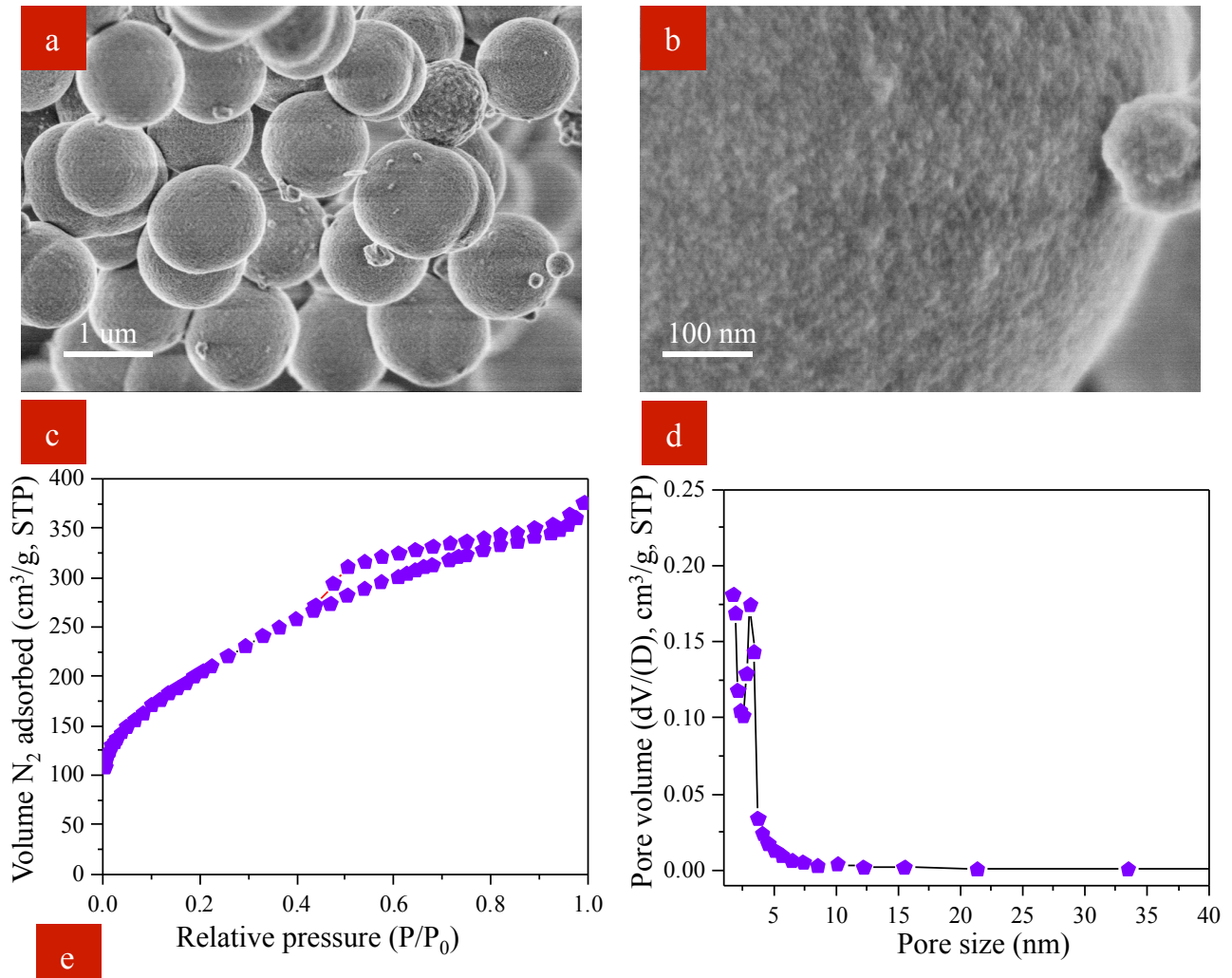

Figure S10. (a, b) SEM images of pure $\mathrm{SiO}_{2}$ which was synthesized using the same method, and the corresponding (c) nitrogen sorption-desorption isotherm, (d) pore size distribution and (e) microstructure analysis. 
Table S1. Comparison of battery performance with various sulfur cathode materials.

\begin{tabular}{|c|c|c|c|c|c|}
\hline & $\begin{array}{c}\text { Sulfur } \\
\text { Loadings } \\
\left(\mathrm{mg} / \mathrm{cm}^{2}\right) \\
\end{array}$ & Scalability & Cycle number & $\begin{array}{l}\text { Capacity } \\
(\mathrm{mAh} / \mathrm{g})\end{array}$ & $\begin{array}{c}\text { Gravimetric } \\
\text { energy density } \\
(\mathrm{Wh} / \mathrm{kg})\end{array}$ \\
\hline $\mathrm{Si}$ (lithiated)-S full cell ${ }^{1}$ & NA & NA & 100 & $330(0.1 \mathrm{C})$ & $590(0.1 C)$ \\
\hline $\begin{array}{l}\text { Graphite-S/mesoporous } \\
\text { carbon full cell }{ }^{2}\end{array}$ & NA & NA & 80 & $500(1 \mathrm{C})$ & $1000(1 \mathrm{C})$ \\
\hline $\begin{array}{l}\text { S/hollow carbon } \\
\text { spheres }^{3}\end{array}$ & 1.0 & NA & 200 & $590\left(200^{\text {th }}\right.$ at $\left.3.2 \mathrm{C}\right)$ & $\sim 1239(3.2 \mathrm{C})^{*}$ \\
\hline $\begin{array}{l}\mathrm{S} / \mathrm{Ni} / \text { popcorn inspired } \\
\text { porous carbon }{ }^{4}\end{array}$ & 4.0 & Yes & 300 & $440\left(300^{\text {th }}\right.$ at $\left.0.2 \mathrm{C}\right)$ & $\sim 924(0.2 \mathrm{C})^{*}$ \\
\hline S/carbon nano-fiber ${ }^{5}$ & $4.4-10.5$ & Yes & 100 & $680\left(100^{\text {th }}\right.$ at $\left.0.1 \mathrm{C}\right)$ & $\sim 1428(0.1 \mathrm{C}) *$ \\
\hline $\mathrm{MnO}_{2} / \mathrm{S}^{6}$ & $0.7-1.0$ & NA & $\begin{array}{l}1200- \\
2000\end{array}$ & $380\left(1000^{\text {th }}\right.$ at $\left.0.2 \mathrm{C}\right)$ & $\sim 798(0.2 \mathrm{C})^{*}$ \\
\hline $\mathrm{S} / \mathrm{TiO}_{2} / \mathrm{CNT}^{7}$ & $1.8-2.7$ & NA & 100 & $848\left(100^{\text {th }}\right.$ at $\left.5 \mathrm{C}\right)$ & $\sim 1780(5 \mathrm{C})^{*}$ \\
\hline $\mathrm{S} / 3-\mathrm{D}$ graphene $^{8}$ & 2.36 & NA & 1000 & $670\left(1000^{\text {th }}\right.$ at $\left.2 C\right)$ & $\sim 1407(2 \mathrm{C})^{*}$ \\
\hline \multirow{4}{*}{$\begin{array}{l}\mathrm{S} @ \mathrm{SiO}_{2} @ \mathbf{A C} \\
\text { (This work) }\end{array}$} & \multirow{2}{*}{2.0} & \multirow{4}{*}{ Yes } & \multirow{2}{*}{1000} & $980\left(300^{\text {th }}\right.$ at $\left.0.2 \mathrm{C}\right)$ & $\sim 2058(0.2 \mathrm{C})$ \\
\hline & & & & $680\left(300^{\text {th }}\right.$ at $\left.0.5 \mathrm{C}\right)$ & $\sim 1428(0.5 \mathrm{C})$ \\
\hline & 4.0 & & 700 & 630 (300th at $0.2 \mathrm{C})$ & $\sim 1323(0.2 \mathrm{C})$ \\
\hline & 5.0 & & 300 & $500\left(300^{\text {th }}\right.$ at $\left.0.5 \mathrm{C}\right)$ & $\sim 1050(0.5 \mathrm{C})$ \\
\hline
\end{tabular}

* The gravimetric energy density was calculated based on the active material only at the average operation voltage of $2.1 \mathrm{~V}$ from literatures. 


\section{Supplementary references:}

1. Shen, C. F., Ge, M. Y., Zhang, A. Y., Fang, X., Liu, Y. H., Rong, J. P., Zhou, C. W. Silicon(lithiated)-sulfur full cells with porous silicon anode shielded by Nafion against polysulfides to achieve high capacity and energy density. Nano Energy 19, 68-77 (2016).

2. Agostini, M., Scrosati, B., Hassoun, J. An advanced lithium-ion sulfur battery for high energy storage. Adv. Energy Mater., 5, 1500481 (2015).

3. Sun, Q., He, B., Zhang, X. Q., Lu, A. H. Engineering of hollow core-shell interlinked carbon spheres for highly stable lithium-sulfur batteries. ACS Nano, 9, 8504-8513 (2015).

4. Xia, X. H., Deng, S. J., Zhan, J. Y., Fang, R. Y., Zhong, Y., Xia, Y., Wang, X. L., Zhang, Q., Tu, J. P., Popcorn inspired porous macrocellular carbon: rapid puffing fabrication from rice and its applications in lithium-sulfur batteries. Adv. Energy Mater., 8, 1701110 (2018).

5. Kim, J. H., Kim, D. K., Lee, H. W., Suppressing polysulfide dissolution via cohesive forces by interwoven carbon nanofibers for high-areal-capacity lithium-sulfur batteries. Nano Lett., 18, 475-481 (2018).

6. Liang, X., Hart, C., Pang, Q., Garsuch, A., Weiss, T., Nazar, L. F. A highly efficient polysulfide mediator for lithium-sulfur batteries. Nat. Commun., 6, $5682(2015)$.

7. Kim, H. M., Lee, S. K., Lee, J. H., Abouimrane, A., Hwang, J. Y., Khaleel, M. A., Belharouak, I., Manthiram, A., Sun, Y. K., High-energy, high-rate, lithium-sulfur batteries: synergetic effect of hollow $\mathrm{TiO}_{2}$-webbed carbon nanotubes and a dual functional carbon-paper interlayer. Adv. Energy Mater., 6, 1501480 (2016).

8. Li, G.X., Sun, J. H., Hou, W. P., Jiang, S. D., Huang, Y., Geng, J. X. Threedimensional porous carbon composites containing high sulfur nanoparticle content for high-performance lithium-sulfur batteries. Nat. Commun., 7, 10601 (2016). 